\title{
Механизм солнечной вспышки - результаты МГД-моделирования и наблюдений
}

\author{
А.И. Подгорныцй \\ 1 Физический институт РАН им. П.Н. Лебедева, Ленинский проспект, 53, Москва, Россия \\ podgorny@lebedev.ru \\ 2 Институт астрономии РАН, ул. Пятницкая, 48, Москва, Россия \\ podgorny@inasan.ru
}

Поступила в редакцию 15 ноября 2017 г.

\begin{abstract}
Аннотация. Появление теплового рентгеновского излучения в короне во время солнечных вспышек и другие наблюдения доказывают, что первичное освобождение энергии солнечной вспышки происходит в солнечной короне на высотах 15000-30000 км. Только механизм вспышки, согласно которому происходит освобождение магнитной энергии токового слоя, может объяснить медленное накопление энергии перед вспышкой, а затем ее быстрое освобождение во время вспышки. Совпадение положения источника теплового рентгеновского излучения с положением токового слоя, полученным из МГД-моделирования над реальной активной областью, при котором все условия берутся из наблюдений, является независимым подтверждением механизма освобождения магнитной энергии токового слоя. Результаты МГД-моделирования показали, что физический смысл процессов накопления и быстрого освобождения энергии в токовом слое лучше всего представляют линии, касательные к проекциям векторов магнитного поля на плоскость конфигурации токового слоя. Основываясь на механизме освобождения энергии в токовом слое и используя результаты численного моделирования и наблюдений, предложена электродинамическая модель солнечной вспышки, объясняющая основные вспышечные явления.

SOLAR FLARE MECHANISM: RESULTS OF THE MHD SIMULATION AND OBSERVATIONS, by A.I. Podgorny and I.M. Podgorny. The appearance of thermal X-ray emission in the corona and other observations proves that primordial energy release of the solar flare takes place in the solar corona at the altitudes of $15000-30000 \mathrm{~km}$. Only the flare mechanism based on the current sheet can explain slow energy accumulation before the flare and then fast energy release during the flare. Coincidence of the position of the thermal X-ray emission source with the current sheet position, obtained from the MHD simulation in which all conditions are taken from observations, can be considered as an independent proof of the mechanism of current sheet magnetic energy release. MHD simulation results show that the physical meaning of the flare energy accumulation and rapid release processes is best demonstrated by the lines which are tangential to the projections of magnetic field vectors on the plane of the current sheet configuration. Basing on the mechanism of fast energy release in a current sheet, using the results of the numerical simulation and observations, the electrodynamical model of the solar flare is proposed to explain its main observational manifestations.
\end{abstract}

Ключевые слова: солнечная вспышка, токовый слой, МГД-моделирование 


\section{1 Введение}

Во время солнечной вспышки за несколько десятков минут происходит освобождение $\sim 10^{32}$ эрг магнитной энергии, которая переходит в тепловую энергию нагретой до $\sim 10^{7} \mathrm{~K}$ плазмы, кинетическую энергию корональных выбросов плазмы, энергию ускоренных заряженных частиц (протонов - до $\sim 20$ ГэВ) и энергию излучения в широком диапазоне частот, создаваемого ускоренными и тепловыми частицами - рентгеновское излучение (мягкое тепловое 1-20 кэВ и жесткое пучковое 20-200 кэВ и выше), радиоизлучение, оптическое излучение, ультрафиолетовое излучение, $\gamma$-излучение. Вспышки происходят над активными областями (AO), представляющими собой образования на фотосфере (солнечной поверхности), в которых магнитное поле (до 3000 Гс) значительно увеличено по сравнению с фоновым полем фотосферы $(\sim 1$ Гс). При этом поток магнитного поля АО увеличивается перед вспышкой, достигая $\sim 10^{22}$ Мкс (Подгорный и др., 2015). Первичное освобождение энергии солнечной вспышки происходит в солнечной короне на высотах 15000-30000 км, что доказано экспериментально благодаря наблюдениям с высоким разрешением теплового рентгеновского излучения от вспышек на лимбе (Лин и др., 2003). Наблюдения предвспышечной ситуации и вспышки в спектральных линях ионов высокоионизованного железа (излучающих при температуре 6.3, 10 и 20 MK) показывают эволюцию структуры с высокой температурой, которая происходит в горячей короне перед вспышкой. Вспышечное выделение энергии в короне отчетливо наблюдается в линиях ионов железа для вспышек на лимбе диска. Во время вспышки в АО остаются практически неизменными магнитный поток, распределения нормальной компоненты магнитного поля и компоненты поля вдоль луча зрения (Подгорный и др., 2015). Вспышки появляются прежде всего в тех АО, где полярности магнитного поля сильно перепутаны (признак, используемый для прогноза вспышек), в этом случае в магнитном поле короны могут появляться особые линии Х-типа, в которых под воздействием возмущений образуются токовые слои (ТС).

Для выяснения механизма вспышки проводилось МГД-моделирование над реальной АО, когда все условия берутся из наблюдений, и никаких предположений о механизме вспышки при постановке условий не делалось. Целью такого моделирования является установить механизм вспышки. Предложенный подход отличался от очень распространенного метода, когда с самого начала выдвигалась гипотеза о механизме вспышки, которая затем проверялась.

Численное МГД-моделирование показало, что появление вспышки в короне объясняется накоплением энергии в магнитном поле ТС. Слой образуется в окрестности особой линии магнитного поля $\mathrm{X}$-типа под действием возмущений, распространяющихся от фотосферы. Магнитные силы $\mathbf{j} \times \mathbf{B} / c$ вызывают движение плазмы, которое деформирует магнитное поле в конфигурацию, соответствующую TC. После квазистационарной эволюции, в ходе которой полная масса плазмы слоя уменьшается вследствие быстрого вытекания плазмы из слоя под воздействием силы магнитного натяжения (Подгорный, Подгорный, 2003, 2012a), ТС переходит в неустойчивое состояние, и происходит взрывное освобождение его магнитной энергии (Подгорный, 1989).

Проведенное недавно китайско-американской группой моделирование вспышечной ситуации с использованием суперкомпьютера в близкой к нашей постановке задачи (Джианг и др., 2016) подтвердило наши результаты. В месте вспышечного освобождения энергии появлялся ТС, магнитные линии вблизи него хорошо совпадают с линиями, полученными из изображений ультрафиолетового излучения вблизи места вспышки. При этом появление жгутов или каких-либо других признаков альтернативных механизмов вспышки, как и у нас, обнаружено не было.

В работе (Джианг и др., 2016) изучалась крупная вспышка бала Х3.1, произошедшая 24 октября 2014 года в AO NOAA 12192, размер которой превосходил размеры всех АО за последние 25 лет, так что ТС можно было легко найти непосредственно из рассчитанной конфигурации поля без использования разработанной нами графической системы поиска.

В настоящее время предложен ряд альтернативных механизмов солнечной вспышки, однако кроме механизма освобождения энергии ТС авторам не известны другие механизмы, которые могли бы объяснить медленное накопление энергии в устойчивой конфигурации, а затем ее переход в неустойчивое состояние в условиях реальной АО. Наиболее популярный механизм объясняет вспышку появлением магнитного жгута с током (Форбес, 1991). Согласно этим представлениям, жгут ускоряется вверх 
магнитной силой $\mathbf{j} \times \mathbf{B} / c$, что приводит к выбросу плазмы и вспышечному освобождению энергии. Для моделирования этого процесса чаще всего с самого начала задается неустойчивая или неравновесная конфигурация с магнитным жгутом. Неудачные попытки получить жгут в короне в результате эволюции магнитного поля под действием возмущений на фотосфере проводились в работах (Торок, Клим, 2005; Аулайнер и др., 2010; Зукарелло и др., 2017). В работе (Торок, Клим, 2005) жгут искусственно задавался закручиванием оснований магнитной арки. После закручивания, превосходящего пороговое $(4 \pi)$, возникала изгибная неустойчивость, однако для появления такой ситуации над АО скорость закручивания должна была превосходить на порядок наблюдаемую скорость вращения пятен.

В работах (Аулайнер и др., 2010; Зукарелло и др., 2017) при постановке задачи МГдмоделирования начальное магнитное поле, задаваемое двумя магнитными зарядами, чрезвычайно упрощено по сравнению с полем реальной $\mathrm{AO}$, а движение плазмы на фотосфере, приводящее к требуемой деформации поля в короне, задавалось настолько сложным, что его реализацию трудно себе представить на фотосфере в АО Солнца. Кроме того, для появления жгута в такой ситуации невозможно обойтись без пересоединения, а значит и ТС, без которого пересоединение невозможно. А само появление ТС уже означает возможность вспышки, так что в этом случае для вспышки не нужен жгут. Даже в этой неестественной ситуации, которая непонятно как может быть получена над реальной АО, энерговыделение вспышки должно происходить в ТС.

Согласно механизму (Зайцев и др., 2015), первичное вспышечное освобождение энергии происходит в хромосфере. Вследствие неустойчивости Релея-Тейлора выделяется энергия плазмы в гравитационном поле Солнца, которая первоначально удерживается магнитным полем. В работе Зайцева и др. (2015) не дано ответа, как в реальной АО могла возникнуть ситуация, при которой значительное количество плазмы, обладающей большой массой, оказалось над областью с сильным магнитным полем и разреженной плазмой.

\section{2 Электродинамическая модель солнечной вспышки}

Основываясь на механизме освобождения энергии в ТС, используя результаты численного моделирования и наблюдений, предложена электродинамическая модель солнечной вспышки (рис. 1), объяс-

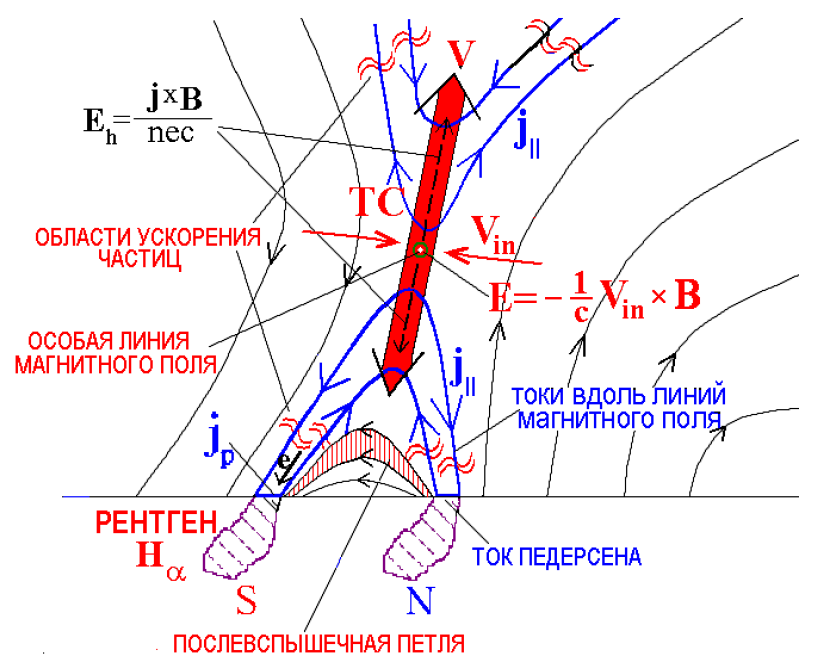

Рис. 1. Электродинамическая модель солнечной вспышки

няющая ее основные наблюдаемые проявления (Подгорный, Подгорный, 2012б). Электрическое поле Лоренца ускоряет протоны вдоль особой линии до энергий $\sim 20$ ГэВ. Ускорение плазмы вдоль слоя 
вверх силой магнитного натяжения, которая значительно увеличивается во время взрывной неустойчивости, вызывает выброс корональной массы. Источники жесткого рентгеновского излучения располагаются в местах пересечения с солнечной поверхностью линий магнитного поля, выходящих из ТС. Рентгеновское излучение появляется в результате взаимодействия электронов, ускоренных в продольных токах, с нижними плотными слоями солнечной атмосферы. Продольные токи вдоль магнитных линий, пересекающих ТС, вызываются электрическим полем Холла, появившимся вследствие ускорения вдоль ТС электронов силой $j B_{n}$ - произведения плотности тока в ТС на компоненту магнитного поля, перпендикулярную слою.

Электроны, ускоренные в продольных токах, вызывают также ряд других наблюдательных процессов вблизи солнечной поверхности - появление лент в излучении $H_{\alpha}$ и $1600 \AA$, а также субтерагерцовое излучение на ленгмюровской частоте. Поскольку некоторые наблюдения излучения, возникающего в хромосфере, проводились когда еще не было наблюдений в рентгеновском и ультрафиолетовом диапазонах, позволивших установить первичное энерговыделение вспышки в короне, появился термин "хромосферная вспышка". Не особенно удачное название "хромосферная вспышка" продолжает изредка использоваться в литературе - имеются в виду процессы в хромосфере, вызванные вспышечным энерговыделением в короне.

\section{3 Постановка задачи МГД-моделирования в короне над АО}

Решение системы МГД-уравнений в солнечной короне проводилось в расчетной области в виде прямоугольного параллелепипеда. Его нижняя граница располагается на фотосфере и представляет собой квадрат размером 400000 км. Центр этого квадрата расположен в середине АО, для АO NOAA 10365 в момент вспышки 02:53 27 мая 2003 г. координаты центра АО на диске Солнца $\left(118.3^{\prime \prime},-87.27^{\prime \prime}\right)$. Измеряемые на фотосфере магнитные поля использовались для задания граничных условий. Условия для задания остальных величин на фотосферной и нефотосферной границах расчетной области аппроксимировались условиями свободного выхода. Решение начинается за несколько дней перед вспышкой, когда нет значительных возмущений в короне, и поэтому потенциальное магнитное поле, рассчитанное по распределению поля на фотосфере, может быть взято в качестве начального условия. Подробно условия моделирования в короне над АО описаны в (Подгорный, Подгорный, 2008). Для повышения скорости расчета авторами специально разработана устойчивая при больших временных шагах конечно-разностная схема (Подгорный, Подгорный, 2004). Схема является абсолютно неявной, противопоточной и консервативной относительно магнитного потока. Разработанные численные методы реализованы в программе “ПЕРЕСВЕТ".

Несмотря на применение специально разработанных методов, МГД-моделирование в короне над AО выполняется настолько медленно, что на обычном компьютере для АО 10365 расчет удалось провести только в сильно сокращенном масштабе времени (магнитное поле в задаваемых на границе условиях менялось в $10^{4}$ раз быстрее, чем на фотосфере Солнца). При таком расчете в результате неестественно быстрого изменения поля у фотосферной границы возникает численная неустойчивость, искажающая решение. Однако применяемые методы дали возможность не допустить распространение неустойчивости внутрь расчетной области от фотосферной границы и ограничить рост величин в приграничной области, так чтобы был возможен расчет. В дальнейшем предполагается провести расчет в реальном масштабе времени на суперкомпьютере, для чего необходимо будет провести распараллеливание вычислений.

\section{4 Конфигурация магнитного поля в месте вспышки и источники теплового рентгеновского излучения}

Максимум плотности тока в ТС для вспышки 27 мая 2003 г. в 02:53 расположен на высоте 16000 км. Его положение в плоскости, перпендикулярной лучу зрения в координатах солнечного диска $(x, y)=\left(96^{\prime \prime}, 56^{\prime \prime}\right)$ (см. рис. $\left.2 \mathrm{a}\right)$, хорошо совпадает с положением максимума интенсивности теплового рентгеновского излучения $(x, y)=\left(99^{\prime \prime}, 64^{\prime \prime}\right)$ (Подгорный, Подгорный, 2013). Небольшое 
отличие может возникнуть из-за неопределенности момента времени при моделировании в сильно сокращенном масштабе времени и вследствие ошибок аппроксимации численного метода.

a

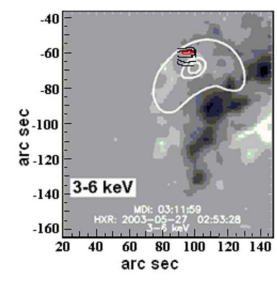

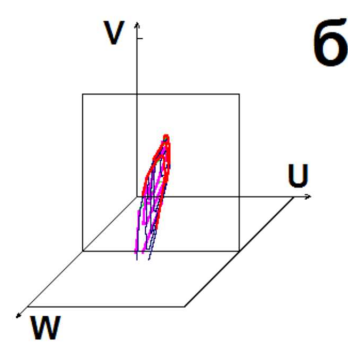

6

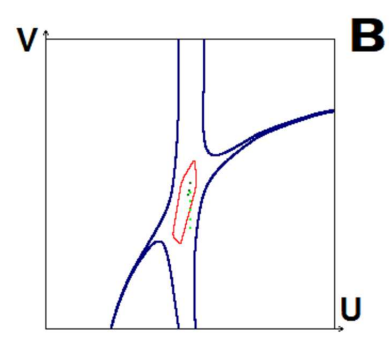

B

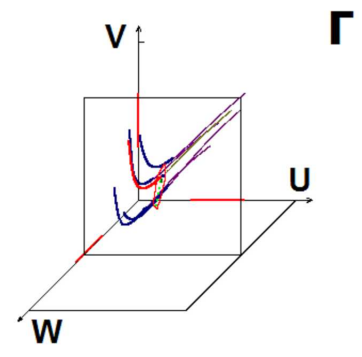

Рис. 2. а - распределение интенсивности теплового рентгеновского излучения для вспышки 27 мая 2003 г. в 02:53, полученное на аппарате RHESSI, наложенное на магнитограмму поля (SOHO/MDI); вблизи найденного положения ТС изображены линии уровня максимальной плотности тока вдоль луча зрения. б - поверхность уровня плотности тока. в - линии, касательные к проекциям векторов магнитного поля на плоскость конфигурации. г - магнитные линии вблизи ТС

Перпендикулярная вектору магнитного поля в точке максимума плотности тока плоскость конфигурации ТС наклонена на $18^{\circ}$ к плоскости, перпендикулярной фотосфере. Поверхность уровня равной плотности тока на рис. 2б дает представление о расположении ТС в пространстве. Конфигурацию магнитного поля, соответствующую ТС, представляют на рис. 2в линии, касательные к проекциям векторов магнитного поля на плоскость конфигурации ТС. Они указывают на направление перпендикулярных им магнитных сил, приводящих плазму в движение - втеканию плазмы в слой в перпендикулярном ему направлении и вытекании вдоль слоя. Эти силы создают ТС с накопленной в нем магнитной энергией. Силы возникают вследствие появления тока, перпендикулярного плоскости. Силы определяются только компонентами поля на плоскости, т. е. проекциями векторов магнитного поля, касательные к которым изображены на рис. 2в.

На рис. 2г представлены магнитные линии, проходящие вблизи центра ТС. Четыре линии проходят через те же точки на плоскости конфигурации, что и касательные к проекциям векторов на рис. 2в, их часть перед плоскостью конфигурации изображена как жирная синяя линия, а за этой плоскостью - тонкая фиолетовая. Еще одна линия проходит через центр слоя, она изображена как жирная красная линия перед плоскостью конфигурации, а за этой плоскостью - тонкая коричневая. Учет всех компонент магнитного поля для магнитной линии делает конфигурацию поля настолько сложной, что в настоящее время трудно установить физический смысл расположения магнитных линий для процессов в ТС.

\section{5 Выводы и задачи для дальнейшего изучения солнечных вспышек}

1. Разработаны методы численного решения МГД-уравнений, предназначенные для моделирования вспышечной ситуации в реальной АО, и графические методы анализа полученных решений.

2. Показано совпадение положения источника теплового рентгеновского излучения с положением ТС, полученным из МГД-моделирования над реальной АО, при котором все условия берутся из наблюдений. Этот результат является независимым подтверждением механизма, согласно которому во время вспышки освобождается магнитная энергия ТС, образовавшегося в короне.

3. Для некоторых вспышек магнитные линии не дают полного преставления о физическом смысле процессов накопления и быстрого освобождения энергии. О процессах в ТС можно судить по 
форме линий в плоскости конфигурации ТС, касательными к проекциям векторов магнитного поля на эту плоскость. Расположение этих линий, имеющих противоположные направления по обе стороны от слоя, соответствует направлению магнитных сил, которые создают ТС, а затем способны его разрушить при переходе системы в неустойчивое состояние.

4. В настоящее время только механизм ТС способен объяснить медленное накопление энергии перед вспышкой, а затем быстрое ее освобождение во время вспышки. При накоплении энергии система должна быть устойчивой. ТС, образующийся в окрестности особой линии магнитного поля Xтипа под действием возмущений, является устойчивым. После квазистационарной эволюции, в ходе которой полная масса плазмы слоя уменьшается, ТС переходит в неустойчивое состояние. Другие механизмы в настоящее время не способны объяснить медленное накопление, за которым следует быстрое освобождение энергии в реальных условиях над АО.

5. Многочисленные наблюдательные данные однозначно указывают на то, что первичное энерговыделение вспышки происходит в нижней короне на высоте 10000-30000 км. Прежде всего, это появление источника теплового рентгеновского излучения вспышки на такой высоте при наблюдениях вспышек на лимбе (Лин и др., 2003). Энерговыделение вспышки в короне подтверждается также наблюдениями в спектральных линях ионов высокоионизованного железа, отсутствием сколько-нибудь значительного изменения магнитного поля на солнечной поверхности во время вспышки, образованием всегда во время вспышки по крайней мере двух лент в излучении $H_{\alpha}$, появлением вспышек прежде всего в тех АО, где полярности магнитного поля сильно перепутаны, что указывает на существование в поле короны особых линий X-типа (признак, используемый для прогноза вспышек).

6. Основываясь на механизме освобождения энергии в ТС и используя результаты численного моделирования, предложена электродинамическая модель солнечной вспышки, объясняющая ее основные наблюдаемые проявления.

7. Несмотря на приведенные сильные аргументы в пользу механизма ТС, объясняющего наблюдаемое появление вспышки в короне, для окончательного решения проблемы выяснения механизма солнечных вспышек, а также, возможно, для улучшения качества прогноза вспышек на основании понимания их механизма, необходимо проведение дополнительных более точных расчетов вспышечной ситуации для многих АО. В настоящее время все методы численного решения и их графического анализа подготовлены.

Авторы благодарны Н.С. Мешалкиной за помощь в нахождении наблюдательных данных.

\section{Литература}

Аулайнер и др. (Aulanier G. et al.) // Astrophys. J. 2010. V. 708. Р. 314.

Джианг и др. (Jiang C. et al.) // Astrophys. J. 2016. V. 828. Issue 1. id. 62.

Зайцев В.В. и др. // XIX Всероссийская ежегодная конференция по физике Солнца "Солнечная и солнечно-земная физика - 2015”. СПб: ГАО РАН. 2015. С. 149.

Зукарелло и др. (Zuccarello F.P. et al.) // Astrophys. J. 2017. V. 837. Issue 2. id. 115.

Лин и др. (Lin R.P. et al.) // Astrophys. J. 2003. V. 595. P. 76.

Подгорный (Podgorny A.I.) // Solar Phys. 1989. V. 123. P. 285.

Подгорный А.И., Подгорный И.М. // Астрон. журн. 2003. Т. 80. С. 753.

Подгорный А.И., Подгорный И.М. // Журн. вычисл. мат. и матем. физ. 2004. Т. 44. С. 1873.

Подгорный А.И., Подгорный И.М. // Астрон. журн. 2008. Т. 85. С. 739.

Подгорный А.И., Подгорный И.М. // Геомагнетизм и аэрономия. 2012а. Т. 52. С. 163.

Подгорный А.И., Подгорный И.М. // Геомагнетизм и аэрономия. 2012б. Т. 52. С. 167.

Подгорный, Подгорный (Podgorny A.I., Podgorny I.M.) // Sun Geosphere. 2013. V. 8. P. 71.

Подгорный А.И. и др. // Астрон. журн. 2015. Т. 92. С. 669.

Торок, Клим (Torok T., Kliem В.) // Astrophys. J. 2005. V. 630. P. L97.

Форбес (Forbes T.G.) // Geophys. Astrophys. Fluid Dyn. 1991. V. 62. P. 16. 